\title{
Editorial: Biomechanics and Mechanobiology
}

\author{
O. Bavi ${ }^{1 \star}$, M. Ozmaeian ${ }^{2}$ and M. H. Heydari ${ }^{3}$ \\ ${ }^{1}$ Department of Mechanical and Aerospace Engineering, Shiraz University of Technology, Shiraz, Iran, ${ }^{2}$ Department of Chemistry, \\ University of Texas at Austin, Austin, TX, United States, ${ }^{3}$ Department of Mathematics, Shiraz University of Technology, Shiraz, Iran
}

Keywords: biomechanics, mechanobiology, axonal injury, digital prototype design, mechanical properties, aging, stent, curved vessel

\section{Editorial on the Research Topic}

\author{
Biomechanics and Mechanobiology
}

As the main subarea of biomechanical engineering, Biomechanics, and Mechanobiology deal with the study of mutual interaction of mechanical forces and structural changes in biomechanical systems from the nanoscale components of living systems to macroscale artificial organs. Simulation, design, material, mathematical modeling, numerical analysis, and experimental verification of mechanical properties and behavior of biomechanical systems are some aspects of this research topic.

In living cells, a precise evaluation of mechanical forces, mechanical properties, and damages of the stimulated cells are the key connection and the most important common denominator of biomechanical/biomedical applications. For example, dealing with the production of surgical ligaments and helmets, the brain tissue behavior and axonal damage extent in different loads, volume fractions, and loading rates should be investigated.

In the first article of our Special Volume, Kazempour et al. present a numerical study of axonal injury in different heterogeneous porcine brain parts with different axon distributions under quasistatic loading. Developing a MATLAB code to create axons in different distributions, generating the coordinates by the Python code as the entry for ABAQUS, and defining an optimal RVE to analyze the visco-hyperelastic model of the tissue, they investigate the sensitivity and vulnerability of tissue to high loading rates. The results of this study are useful in the production of helmets, surgical robots, and all the tools which are used for preventing or treating brain injuries.

Among the various approaches in numerical modeling, the digital prototype is one of the main techniques of calculating the elements of an inhomogeneous structure under the influence of external forces. In the second article, Gerasimov et al. consider a finite element analysis method based on computed tomography data. In their calculations, they use a three-dimensional isoparametric finite element of a continuous medium developed by the authors with a linear approximation based on weighted integration of the local stiffness matrix. The purpose of this study is to describe a general algorithm for constructing a numerical model that allows static calculation of objects with a porous structure according to its computed tomography data.

Due to the key connection of mechanical properties, geometry, and loading mode of bones in ensuring the health and balance of the human body, enormous experimental and computational biomechanical analyses are performed to investigate the effective parameters of these factors. Among these parameters, aging (from 40 to +80 years old) has a dominant effect on geometrical and mechanical properties changes of bones. In the third article of the volume, Sahandifar and Kleiven developed three distinct sets of numerical models to investigate the separated and combined effects of key changes in the geometry and mechanical properties on the strength of the femur in men and women. The results of this study show that the fracture forces reduced in both men and women when 
accounting for both mechanical and geometrical changes due to aging. The geometrical changes due to aging counteracted some of the adverse effects of the mechanical changes in men, whereas it adversely affected the fracture forces in women.

In the fourth article, using the finite element method, Baradaran et al. designed a special stent that can be implanted in a curved vessel. The design has been performed based on the appropriate flexibility and radial strength design, depending on the characteristics of a particular case study. In the optimization procedure, flexibility and radial strength have been evaluated based on ASTM standard mechanical tests. The main contribution of this work is the use of spline curvature point coordinates and stent thickness to considerably expand the range of stent shapes that a single geometric modeler can produce. Proposing a method to use spline point coordinates and strut thickness as optimization design variables, a remarkable geometric variation compared to previous stent parameterizations was achieved. The proposed model can also be used to simulate stents with other elastoplastic materials as well as other applications such as biliary stent which is used to allow the flow of urine between the kidney and the bladder.

Finally, in the fifth article, Dehparvar et al. are focused on prosthetic heart valves. These valves are commonly used as a treatment for aortic valve deficiencies. They discuss the effects of leaflet design on the performance of the valve by performing fully coupled fluid-structure interaction (FSI) analyses of opening and closing of prosthetic heart valves with various leaflet designs. This study shows that the leaflets with a linear profile and larger slope at the skirt area give the required features. So, constructing a $3 \mathrm{D}$ model by variable geometrical parameters and carrying out fully-coupled FSI analysis, they have claimed that the present model gives better results for valve opening area and the stress developed on the leaflets.

\section{AUTHOR CONTRIBUTIONS}

This Editorial was prepared jointly by $\mathrm{OB}, \mathrm{MO}$, and $\mathrm{MH}$.

Conflict of Interest: The authors declare that the research was conducted in the absence of any commercial or financial relationships that could be construed as a potential conflict of interest.

Publisher's Note: All claims expressed in this article are solely those of the authors and do not necessarily represent those of their affiliated organizations, or those of the publisher, the editors and the reviewers. Any product that may be evaluated in this article, or claim that may be made by its manufacturer, is not guaranteed or endorsed by the publisher.

Copyright (C) 2022 Bavi, Ozmaeian and Heydari. This is an open-access article distributed under the terms of the Creative Commons Attribution License (CC BY). The use, distribution or reproduction in other forums is permitted, provided the original author(s) and the copyright owner(s) are credited and that the original publication in this journal is cited, in accordance with accepted academic practice. No use, distribution or reproduction is permitted which does not comply with these terms. 